\title{
Factors associated with delayed emergency room visits in adult immigrant patients with mild abdominal pain in Korea
}

\author{
Do Young Kim', Dae Hee Kim², Hai-Jeon Yoon ${ }^{3}$, Woon Jeong Lee', \\ Seon Hee $\mathrm{Woo}^{1}$, Seung Hwan Seol ${ }^{2}$, Han Joon $\mathrm{Kim}^{1}$ \\ 'Department of Emergency Medicine, The Catholic University of Korea College of Medicine, Seoul, Korea \\ ${ }^{2}$ Department of Emergency Medicine, Incheon St. Mary's Hospital, The Catholic University of Korea College \\ of Medicine, Seoul, Korea \\ ${ }^{3}$ Department of Nuclear Medicine, Ewha Womans University College of Medicine, Seoul, Korea
}

Objective To determine the factors associated with unmet needs in immigrant patients complaining of abdominal pain, by analyzing those associated with the time from symptom onset to emergency room visit.

\begin{abstract}
Methods We retrospectively reviewed the medical records of immigrants with abdominal pain who visited a tertiary hospital emergency department from January to December 2016. The dependent variable was the time from symptom onset to emergency room visit. The independent variables were age, sex, vital signs, disposition, health insurance status, date of visit, time of visit, level of education, employment status, economic satisfaction, marital status, living with family, duration of residence, having a native spouse, and subjective proficiency in Korean. We analyzed the association of the dependent variable with each independent variable.
\end{abstract}

Results In total, 102 immigrant patients with abdominal pain were enrolled in this study. The patients who had earlier visits had good subjective proficiency in Korean, high economic satisfaction, longer durations of residence, a tendency to have a native spouse, and a high employment rate. After linear regression analysis, the time from symptom onset to emergency room visit was negatively associated with employment (adjusted odds ratio, $-13.67 ; 95 \%$ confidence interval, -23.25 to $-4.09 ; \mathrm{P}=0.006$ ) and having a native spouse (adjusted odds ratio, $-11.7 ; 95 \%$ confidence interval, -20.61 to $-2.8 ; \mathrm{P}=0.011$ ).

Conclusion The factors influencing the time from symptom onset to emergency room visit in immigrant patients with abdominal pain are associated with social capital, which improves access to emergency care. Policies that improve immigrant access to emergency care should be considered.

Keywords Emigrants and immigrants; Emergencies; Health services needs and demand
elSSN: 2383-4625

Received: 7 April 2018

Revised: 21 June 2018

Accepted: 26 June 2018

Correspondence to: Dae Hee Kim Department of Emergency Medicine, Incheon St. Mary's Hospital, The Catholic University of Korea College of Medicine, 56 Dongsu-ro, Bupyeong-gu, Incheon 21431, Korea

E-mail: kim_dae_hee@catholic.ac.kr ORCID

http://orcid.org/0000-0001-5104-9806

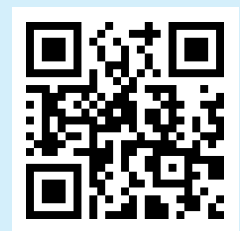

How to cite this article:

Kim DY, Kim DH, Yoon HJ, Lee WJ, Woo SH, Seol SH, Kim HJ. Factors associated with delayed emergency room visits in adult immigrant patients with mild abdominal pain in Korea. Clin Exp Emerg Med 2019;6(2):138-143.

This is an Open Access article distributed under the terms of the Creative Commons Attribution Non-Commercial License (http:// creativecommons.org/licenses/by-nc/4.0/). 


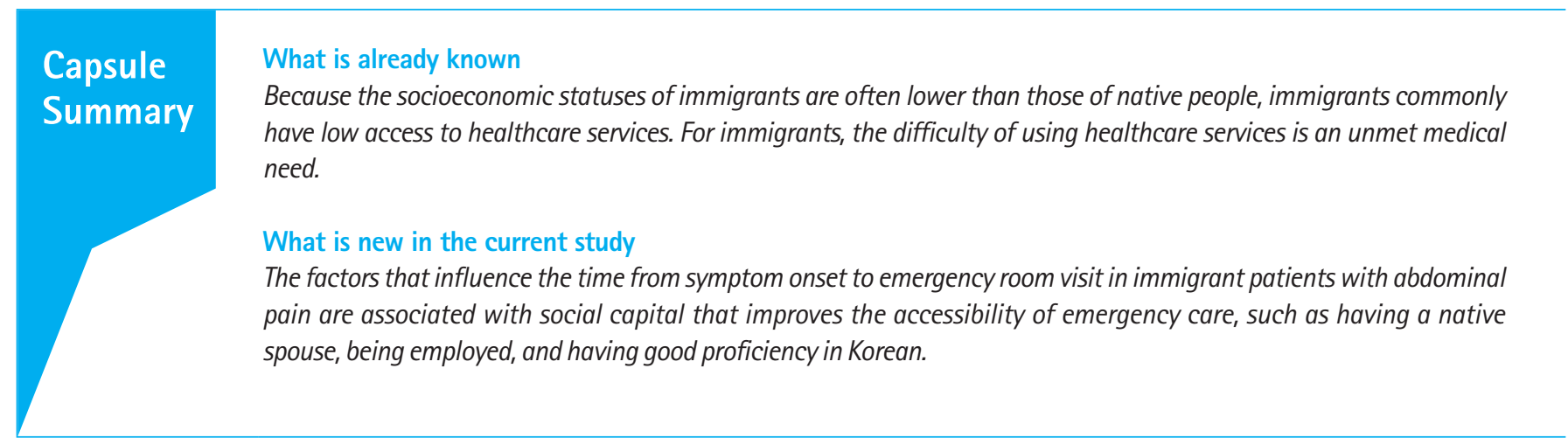

\section{INTRODUCTION}

The immigrant population continues to increase in many developed countries, but providing adequate healthcare services to immigrants remains a challenging task. ${ }^{1}$ This difficulty reflects the special circumstances of immigrants, who lack language communication skills, have limited knowledge of community resources, and sometimes have legal restrictions. ${ }^{2}$ Because the socioeconomic status of immigrants is often lower than that of native people, immigrants commonly have low access to healthcare services. ${ }^{3-6}$ For a minority, the difficulty of using healthcare services is one unmet medical need, which is defined as the difference between healthcare services that are deemed necessary to properly deal with a health problem and the healthcare service used. $^{7.8}$

Married immigrants and their children in Korea are not significantly different from those elsewhere, and they have low access to healthcare services. ${ }^{9}$ However, there have been no studies on the factors associated with low emergency care accessibility for immigrants. The length of the pre-hospital interval can be affected by the socioeconomic factors of the patient, along with factors related to emergency medical services in Pakistan. ${ }^{10}$ These factors are also associated with low emergency care accessibility in the United States. ${ }^{11}$ Therefore, the purpose of this study is to determine the factors associated with low emergency care accessibility by analyzing the factors associated with the symptom onset to emergency room visit time in immigrant patients complaining of abdominal pain.

\section{METHODS}

\section{Study population}

According to the 2016 Population and household census released by Statistics Korea, as of November 1, the number of immigrants in Korea was $1,414,000$, which accounts for $2.8 \%$ of the total population and an increase of 50,000 from the previous year. Of the immigrants in Korea, 880,000 (62.2\%) lived in the Seoul metropolitan area and accounted for $3.5 \%$ of the inhabitants of this area. By nationality, the number of Chinese citizens was the highest, at 692,000 (49\%), followed by Vietnamese citizens 133,000 (9.4\%). By age, the number of foreign residents between the ages of 20 and 29 was the highest, at 391,000 (27.7\%), followed by those between the ages of 30 and 39, at 364,000 (25.7\%). ${ }^{12}$

This study was conducted at a medical college-affiliated hospital in Incheon, Korea. Incheon is a city located in the Seoul metropolitan area and the third largest city in Korea, with a population of 2.91 million. Incheon has 91,525 immigrants, which comprise $3.16 \%$ of the total Incheon population. ${ }^{12}$ The hospital where the study was conducted is a tertiary referral hospital. In 2016, 57,521 people visited the emergency room and 8,782 (15.3\%) were admitted, of whom 2,007 (3.5\%) were admitted to the intensive care unit.

\section{Study design}

This was a retrospective study. Since 2015, our hospital has used medical records that include a detailed history of socioeconomic factors for immigrant patients, and the study was based on these medical records. This study was designed to minimize the impact of medical factors among those associated with delays in visiting the emergency room for immigrant patients. For this reason, we selected immigrant patients with the same medical condition. Acute abdominal pain, known as one of the most common reasons for visiting the emergency room, was selected as the medical condition. ${ }^{13}$ We also selected immigrant patients with the same severity of acute abdominal pain, using the Korean Triage and Acuity Scale (KTAS) score as the criterion. ${ }^{14}$ Immigrant patients with a KTAS score of 4 were selected for this study.

The study period was 12 months, from January 1 to December 
31, 2016. Patients who were registered with acute abdominal pain in the triage system were identified through the electronic medical record system. From the collected patient list, only patients with a resident registration number beginning with five or six, which refers to immigrants, were considered for inclusion. Among these immigrant patients, only those over the age of 20 were selected as study subjects. Patients were excluded from this study who visited the hospital periodically due to chronic disease, revisited the emergency room due to acute abdominal pain, transferred from other hospital, or had a final diagnosis that was not a gastrointestinal tract-related disease. Patients with chronic disease were defined as patients who visited more than six times during the study period. Revisited patients were defined as patients who visited due to the same abdominal pain within one week.

We set the time from symptom onset to emergency room visit as the dependent variable. Symptom onset was defined as the first occurrence of abdominal pain. The time was calculated based on the patient's statement. The independent variables that were investigated in the analysis were age, sex, vital signs, disposition, health insurance status, date of visit, time of visit, level of education, employment status, economic satisfaction, marital status, living with family, duration of residence, having a native spouse, and subjective proficiency in Korean. We analyzed the association of the dependent variable with each independent variable.

Subjective health status, economic satisfaction, and subjective proficiency in Korean were rated on a five-point scale. The day of visit was categorized as a weekday or a weekend visit. The time of visit was categorized as during work hours (8 a.m. to 6 p.m.) or during off-duty hours ( 6 p.m. to 8 a.m.). The level of education was designated as high school graduate and below or college graduate and above. The duration of residence was defined as the duration from the first year of residence to the present. A native spouse was defined as a Korean citizen born and raised in Korea.

\section{Statistical analysis}

Statistical analyses were performed with PASW Statistics ver. 18.0 (SPSS Inc., Chicago, IL, USA). The difference between the two groups, divided by the median time from symptom onset to emergency room visit, was analyzed by the following statistical analysis methods. Nominal variables were analyzed with the Mann-Whitney U-test, and continuous variables were analyzed with correlation coefficients. Linear regression analysis was also performed to determine the association of each factor with the dependent variable. The factors used in the regression model were those that differed significantly in the comparisons between the two groups. Statistical significance was defined as a P-value less than 0.05 .

\section{Ethical considerations}

The collected data were used for study purposes only. Because this study was a retrospective chart review, informed consent was waived. This study was approved by the institutional review board of Incheon St. Mary's Hospital, College of Medicine, The Catholic University of Korea, Seoul, Republic of Korea (OC17RESI0113v).

\section{RESULTS}

A total of 1,201 immigrant patients visited the emergency room during the study period. The five most frequent symptoms were fever (218 patients, 18.2\%), abdominal pain (157 patients, 13.1\%), dyspnea (149 patients, 12.4\%), headache (102 patients, $8.5 \%$ ), and chest discomfort (82 patients, 6.8\%). Among the pa-

Table 1. Characteristics of immigrant patients with abdominal pain

\begin{tabular}{|c|c|c|c|c|}
\hline & KTAS $2(n=16)$ & KTAS $3(n=34)$ & KTAS $4(n=107)$ & P-value \\
\hline \multicolumn{5}{|l|}{ Sex } \\
\hline Female & 11 & 20 & 72 & 0.638 \\
\hline Male & 5 & 14 & 35 & \\
\hline Age & $33.5(29.75-50.5)$ & $30.5(26-39)$ & $34(28-53)$ & 0.302 \\
\hline Transferred & 3 & 2 & 1 & \\
\hline Chronic disease & 0 & 4 & 0 & \\
\hline Revisited & 1 & 0 & 4 & \\
\hline NRS & $9.5(8.25-10)$ & $7(6-7)$ & $5(4-6)$ & $<0.001$ \\
\hline \multicolumn{5}{|l|}{ Final diagnosis } \\
\hline Gastrointestinal tract-related disease & 2 & 7 & 106 & \\
\hline Female reproductive system disease & 3 & 12 & 1 & \\
\hline Urinary tract-related disease & 10 & 8 & 0 & \\
\hline Cancer & 0 & 4 & 0 & \\
\hline Other disease & 1 & 3 & 0 & \\
\hline
\end{tabular}

Values are presented as number or median (interquartile range).

KTAS, Korean Triage and Acuity Scale; NRS, numeric pain rating scale. 
Table 2. Median time from symptom onset to emergency room visit by demographic and clinical factors

\begin{tabular}{lccc}
\hline & Number & Median time (hr) & P-value \\
\hline Sex & & & \\
Female & $68(66.7)$ & $7.02(2.61-18.98)$ & 0.812 \\
Male & $34(33.3)$ & $10.25(1.08-30)$ & \\
Age (yr) & & & \\
$20-64$ & 95 & 0.269 \\
$\geq 65$ & 7 & \\
Symptoms other than abdominal pain & & \\
Vomiting & & \\
$\quad$ Present & 31 & \\
$\quad$ Absent & 71 & \\
Diarrhea & & \\
$\quad$ Present & & \\
$\quad$ Absent & 24 & \\
Fever & 78 & \\
$\quad$ Present & & \\
$\quad$ Absent & 17 & \\
\end{tabular}

Values are presented as number (\%), median (interquartile range), or number.

tients with abdominal pain, no patients had KTAS scores of 1 and 5. The baseline characteristics of immigrant patients with abdominal pain are shown in Table 1.

Among the 157 patients with abdominal pain, 55 met the exclusion criteria; thus, 102 immigrants with abdominal pain were enrolled in this study. The 55 patients included 50 whose KTAS score was not 4, 4 who revisited due to the same abdominal pain, and 1 whose final diagnosis was not a gastro-intestinal tract-related disease. Of the study subjects, 68 (66.7\%) were female, the mean age was $40.57 \pm 15.06$ years, and the mean duration of residence was $6.99 \pm 5.55$ years. The median time from symptom onset to emergency room visit was 7.36 (2 to 23.85) hours. The most common final diagnosis was acute enteritis, which affected 28 patients (27.5\%).

The differences in the time from symptom onset to emergency room visit were analyzed according to nominal variables. Patients with diarrhea tended to have a shorter time from symptom onset to emergency room visit (Table 2), while admitted patients tended to have a longer time. In contrast, patients who were highly educated, employed, and living with a native spouse tended to have a shorter time from symptom onset to emergency room visit (Table 3).

The correlations between the time from symptom onset to emergency room visit and the continuous variables were analyzed. Vital signs, including systolic blood pressure, diastolic blood pressure, heart rate, respiratory rate, and body temperature, were evaluated as independent variables. There were no significant correlations between the vital signs and the time from symptom onset to emergency room visit. Socioeconomic factors were also evaluated in the correlation analysis, including the duration of
Table 3. Median time from symptom onset to emergency room visit by socio-economic capital

\begin{tabular}{|c|c|c|c|}
\hline & Number & Median time (hr) & P-value \\
\hline \multicolumn{4}{|l|}{ Disposition } \\
\hline Admission & $22(21.6)$ & $1.72(4.76-29.74)$ & 0.031 \\
\hline Discharge & $80(78.4)$ & $5.6(1.71-23.57)$ & \\
\hline \multicolumn{4}{|l|}{ Health insurance } \\
\hline Beneficiary & $71(69.6)$ & $8.2(1.8-24)$ & 0.962 \\
\hline Non-beneficiary & $31(30.4)$ & $7.32(2.06-26.45)$ & \\
\hline \multicolumn{4}{|l|}{ Day of visit } \\
\hline Weekday visits & $57(55.9)$ & $5.68(2-24.18)$ & 0.442 \\
\hline Weekend visits & $45(44.1)$ & $8.62(2.15-24.5)$ & \\
\hline \multicolumn{4}{|l|}{ Time of visit } \\
\hline 8 a.m. to 6 p.m. & 35 (34.3) & $12.68(3.33-25.02)$ & 0.135 \\
\hline 6 p.m. to 8 a.m. & $67(65.7)$ & $5.43(1.8-23.2)$ & \\
\hline \multicolumn{4}{|l|}{ Level of education } \\
\hline College graduates or above & $28(27.5)$ & $4.7(1.16-12.73)$ & 0.032 \\
\hline High school graduates or below & $74(72.5)$ & $8.41(2.98-27.29)$ & \\
\hline \multicolumn{4}{|l|}{ Employment status } \\
\hline Employed & $38(37.3)$ & $3.94(1-12.4)$ & 0.001 \\
\hline Unemployed & $64(62.7)$ & $13.13(3.33-36)$ & \\
\hline \multicolumn{4}{|l|}{ Marriage } \\
\hline Married & $79(77.5)$ & $7.05(2-24)$ & 0.962 \\
\hline Unmarried & $23(22.5)$ & $8.2(2-25.92)$ & \\
\hline \multicolumn{4}{|l|}{ Family living together } \\
\hline Yes & 89 (87.3) & $7.4(2.45-25.47)$ & 0.141 \\
\hline No & $13(12.7)$ & $5.75(0.94-13.35)$ & \\
\hline \multicolumn{4}{|l|}{ Native spouse } \\
\hline Yes & $32(31.4)$ & $3.5(1.43-8.88)$ & 0.003 \\
\hline No & $70(68.6)$ & $12.49(3.05-27.28)$ & \\
\hline
\end{tabular}

Values are presented as number (\%) or median (interquartile range).

residence, subjective proficiency in Korean, and economic satisfaction. All three variables correlated negatively with the time from symptom onset to emergency room visit (Table 4).

A regression analysis was performed with the nominal variables that differed significantly and the continuous variables that exhibited significant correlations. The variables associated with the time from symptom onset to emergency room visit were having a native spouse, being employed, and having good proficiency in Korean (Table 5).

\section{DISCUSSION}

This was the first study of the factors associated with unmet emergency care needs in immigrants. There have been a few previous studies of factors associated with unmet needs for general healthcare services in immigrants, ${ }_{1}^{2,3,6}$ but none have focused on emergency care. Therefore, we sought to determine the factors associated with unmet general healthcare service needs in immigrants by analyzing the time from symptom onset to emergency room visit in immigrants with abdominal pain in Korea.

We retrospectively reviewed the medical records of immigrants 
Table 4. Significant correlation coefficients between the time from symptom onset to emergency room visit and continuous variables

\begin{tabular}{lcccc}
\hline & $\begin{array}{c}\text { Time from symptom onset } \\
\text { to emergency room visit }\end{array}$ & $\begin{array}{c}\text { Duration of } \\
\text { residence }\end{array}$ & $\begin{array}{c}\text { Subjective proficiency } \\
\text { in Korean }\end{array}$ & $\begin{array}{c}\text { Economic } \\
\text { satisfaction }\end{array}$ \\
\hline Time from symptom onset to emergency room visit & 1 & - & - & - \\
Duration of residence & $-0.21^{*}$ & 1 & 1 & - \\
Subjective proficiency in Korean & $-0.05^{* *}$ & -0.11 & $-0.21^{*}$ & - \\
Economic satisfaction & $-0.45^{* *}$ & -0.13 & 1
\end{tabular}

${ }^{*} \mathrm{P}<0.05,{ }^{* * *} \mathrm{P}<0.001$.

Table 5. Multivariable linear regression analysis

\begin{tabular}{lcc}
\hline & aOR $(95 \% \mathrm{Cl})$ & P-value \\
\hline Employed status & $-13.67(-23.25$ to -4.09$)$ & 0.006 \\
Existence of native spouse & $-11.7(-20.61$ to -2.8$)$ & 0.011 \\
Presence of diarrhea & $-3.56(-13.54$ to 6.42$)$ & 0.481 \\
Subjective proficiency in Korean & $-2.38(-5.78$ to 1.04$)$ & 0.171 \\
Economic satisfaction & $-2.03(-6.68$ to 2.62$)$ & 0.388 \\
Residence time & $-0.32(-1.1$ to 0.47$)$ & 0.427 \\
College graduates or above & $0.98(-8.73$ to 10.68$)$ & 0.842 \\
Admission & $1.03(-8.98$ to 11.03$)$ & 0.839 \\
\hline
\end{tabular}

$\mathrm{aOR}$, adjusted odds ratio; $\mathrm{Cl}$, confidence interval.

with abdominal pain who visited a tertiary hospital emergency department. Our results demonstrated that having a native spouse, being employed, and having good proficiency in Korean were associated with the time from symptom onset to emergency room visit in immigrants with abdominal pain in Korea. Thus, the unmet emergency care needs in immigrants are less likely to be associated with medical factors and more likely to be associated with social capital.

The proportion of women among the study subjects (66.7\%) was much higher than the proportion of men (33.3\%). This result was probably associated with differences in accessibility between male and female immigrants. Those who immigrate to Korea for employment are known to feel a heavy burden of medical expenses while using the emergency room. ${ }^{15}$ In other words, male immigrants, who make up a high proportion of those who immigrate for employment, are less likely to visit the emergency room, even if they have medical needs. For the same reason, female immigrants, who make up a high proportion of those who immigrate for marriage, were more likely to be included as study subjects. Therefore, further studies are needed that adjust for differences in sex and accessibility.

Among the nominal variables, those that were associated with significant differences in the dependent variable were disposition, level of education, employment status, and having a native spouse. The admitted group had a longer pre-hospital interval than the non-admitted group. Patients who had longer pre-hospital intervals could be considered to have visited the emergency room after disease progression. On the other hand, the variables related to social capital were associated with a shorter pre-hospital time interval in the capital-rich group. Thus, patients with sufficient social capital could be considered to have visited the emergency room relatively early, using their social capital.

Among the continuous variables, those that correlated significantly with the dependent variable were the duration of residence, subjective proficiency in Korean, and economic satisfaction. The variables related to social capital correlated negatively with the dependent variable. These results also indicate that patients with sufficient social capital may have visited the emergency room relatively early using their social capital.

The variables that were significant in the linear regression analysis were being employed and having a native spouse. Among the variables related to social capital, those that can substantially improve cultural and linguistic accessibility were significant. In other words, the improvement of accessibility using social capital was associated with a reduced pre-hospital time interval.

This study had several limitations. First, because this study included only patients with abdominal pain, it is difficult to generalize the results to patients who visit the emergency room due to trauma or other diseases. Second, an analysis of specific medical factors, such as objective severity and prognosis, was not performed. However, we estimate that the association of medical factors with the pre-hospital time interval is not large. Third, the analysis of accurate economic statuses, such as the income and assets, was not possible because these are considered sensitive personal information and are not collected during history taking. Fourth, this was a retrospective study that was based on the analysis of medical records. In retrospective studies, experimental design and statistical analyses cannot always completely control for confounders.

In conclusion, the factors associated with the time from symptom onset to emergency room visit in immigrant patients with abdominal pain are related to social capital that can improve the accessibility of emergency care, such as having a native spouse, being employed, and having good proficiency in Korean. Policies to improve access to emergency care for immigrant patients should be considered. 


\section{CONFLICT OF INTEREST}

No potential conflict of interest relevant to this article was reported.

\section{REFERENCES}

1. Howe Hasanali S. Immigrant-native disparities in perceived and actual met/unmet need for medical care. J Immigr Minor Health 2015;17:1337-46.

2. DuBard CA, Gizlice Z. Language spoken and differences in health status, access to care, and receipt of preventive services among US Hispanics. Am J Public Health 2008;98:2021-8.

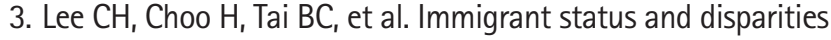
in health care delivery in patients with myocardial infarction. Int J Cardiol 2013;166:696-701.

4. Calvasina $P$, Muntaner $C$, Quinonez $C$. Factors associated with unmet dental care needs in Canadian immigrants: an analysis of the longitudinal survey of immigrants to Canada. BMC Oral Health 2014;14:145.

5. Wong ST, Homma Y, Johnson JL, Saewyc E. The unmet health needs of East Asian high school students: are homestay students at risk? Can J Public Health 2010;101:241-5.

6. Schoevers MA, van den Muijsenbergh ME, Lagro-Janssen AL. Illegal female immigrants in The Netherlands have unmet needs in sexual and reproductive health. J Psychosom Obstet Gynaecol 2010;31:256-64.

7. Giblon R, Bauer GR. Health care availability, quality, and un- met need: a comparison of transgender and cisgender residents of Ontario, Canada. BMC Health Serv Res 2017;17:283.

8. Carr W, Wolfe S. Unmet needs as sociomedical indicators. Int J Health Serv 1976;6:417-30.

9. Lee SH, Park YC, Hwang J, Im JJ, Ahn D. Mental health of intermarried immigrant women and their children in South Korea. J Immigr Minor Health 2014;16:77-85.

10. Noorani MM, Khaliq MF, Shoaib M, et al. Time intervals and associated factors of emergency treatment: first insight into Pakistani system. Int Arch Med 2014;7:41.

11. Wang Y, Wilson FA, Stimpson JP, et al. Fewer immigrants have preventable ED visits in the United States. Am J Emerg Med 2018;36:352-8.

12. Statistics Korea. 2016 Population and housing census. Daejeon: Statistics Korea; 2016.

13. Gerhardt RT, Nelson BK, Keenan S, Kernan L, MacKersie A, Lane MS. Derivation of a clinical guideline for the assessment of nonspecific abdominal pain: the Guideline for Abdominal Pain in the ED Setting (GAPEDS) Phase 1 Study. Am J Emerg Med 2005;23:709-17.

14. Kim JH, Kim JW, Kim SY, et al. Validation of the Korean triage and acuity scale compare to triage by emergency severity index for emergency adult patient: preliminary study in a tertiary hospital emergency medical center. J Korean Soc Emerg Med 2016;27:436-41.

15. Jeon $\mathrm{H}_{\text {, Kang }} \mathrm{GH}$, Jang $\mathrm{YS}$, et al. Evaluation of emergency care for foreign patients in Korea. J Korean Soc Emerg Med 2011; 22:735-42. 